\title{
On the Linear Complexity of Products of Shift-Register Sequences
}

\author{
Rainer Göttfert and Harald Niederreiter \\ Institute for Information Processing \\ Austrian Academy of Sciences \\ Sonnenfelsgasse 19 \\ A-1010 Vienna, Austria \\ E-mail: goet@qünfo.oeaw.ac.at \\ nied@qiinfo.oeaw.ac.at
}

\begin{abstract}
In the theory of stream ciphers the termwise product of shiftregister sequences plays a crucial role. In this paper we improve and generalize earlier results on the linear complexity of a termwise product of two shift-register sequences and we also provide information on the minimal polynomial of such a product.
\end{abstract}

\section{Introduction}

A fundamental problem in the theory of stream ciphers is the determination of the linear complexity of keystreams. A detailed account of this problem can be found in the survey article of Rueppel [8]. In practical implementations, the basic ingredients of typical algorithms for keystream generation are shift-register sequences and Boolean combining functions. It is essential for the analysis of such algorithms to investigate the behavior of shift-register sequences under elementary operations such as termwise addition and multiplication. If this behavior is known, then the effect of general combining functions can also be predicted. Since the sum of shift-register sequences is comparatively easy to analyze, research has focused on the linear complexity of the product of shiftregister sequences. It suffices, of course, to treat the product of two shift-register sequences, as we can then proceed by induction to obtain information about the product of any finite number of shift-register sequences. For prior work on the linear complexity of products of shift-register sequences we refer e.g. to [1], [3], [9], [10]. The basic result in this area says that the linear complexity of a product of shift-register sequences is bounded from above by the product of the linear complexities of the individual sequences (this result is implicit in [10] and stated explicitly in [3, Corollary 3.5]). In this paper we improve and generalize earlier results on the linear complexity of a product of two shift-register sequences and we also provide information on the minimal polynomial of such a product. 
For an arbitrary field $F$ and a nonconstant monic polynomial $f \in F[x]$ let $S_{F}(f)$ be the set of all (linear feedback) shift-register sequences in $F$ with characteristic polynomial $f$. For the constant polynomial $f=1, S_{F}(f)$ consists by definition of the zero sequence, i.e., the sequence all of whose terms are 0 . If $f \in F[x]$ is any monic polynomial, then $S_{F}(f)$ is a vector space over $F$ of dimension $\operatorname{deg}(f)$ under termwise operations on sequences. If a shift-register sequence $\sigma$ in $F$ belongs to $S_{F}(f)$, but to no space $S_{F}(g)$ with $g$ being a proper factor of $f$, then $f$ is called the minimal polynomial of $\sigma$ and its degree $\operatorname{deg}(f)$ is the linear complexity of $\sigma$. We write $M_{F}(f)$ for the subset of $S_{F}(f)$ consisting of the sequences with minimal polynomial $f$.

For arbitrary sequences $\sigma_{1}, \ldots, \sigma_{k}$ of elements of $F$ we define $\sigma_{1}+\cdots+\sigma_{k}$ to be the sequence which is the termwise sum, and for arbitrary sequences $\sigma$ and $\tau$ of elements of $F$ we let $\sigma \tau$ be the sequence which is the termwise product. Thus, for instance, if $\sigma=\left(s_{n}\right)_{n=0}^{\infty}$ and $\tau=\left(t_{n}\right)_{n=0}^{\infty}$, then $\sigma \tau=\left(s_{n} t_{n}\right)_{n=0}^{\infty}$, where all $s_{n}, t_{n} \in F$.

\section{Results and Proofs}

For cryptologic purposes, the only fields $F$ of interest are finite fields, but it should be pointed out that most of our results have analogs for arbitrary fields (see Göttfert [2]). Throughout this paper, $\mathbb{F}_{q}$ denotes a fixed finite field of order $q$ and characteristic $p$. If $F=\mathbb{F}_{q}$, we write $S(f)$ and $M(f)$ for $S_{F}(f)$ and $M_{F}(f)$, respectively. We refer to [4, Chapter 6] for background information on shift-register sequences in finite fields. The following theorem is our main result. There is no serious loss of generality in considering only spaces $S(f)$ with $f(0) \neq 0$ (compare with $[4$, p. 222]).

Theorem 1. Let $f, g \in \mathbb{F}_{q}[x]$ be nonconstant monic polynomials with $f(0) \neq 0$ and $g(0) \neq 0$. Let $\alpha_{1}, \ldots, \alpha_{r}$ be the distinct roots of $f$ in the splitting field $E$ of $f g$ over $\mathbb{F}_{q}$, with corresponding multiplicities $a_{1}, \ldots, a_{r}$. Similarly, let $\beta_{1}, \ldots, \beta_{s} \in$ $E$ be the distinct roots of $g$ with multiplicities $b_{1}, \ldots, b_{s}$, respectively. Suppose that the following two conditions hold:

(i) the rs elements $\alpha_{i} \beta_{j} \in E, 1 \leq i \leq r, 1 \leq j \leq s$, are distinct;

(ii) $\left(\begin{array}{c}a_{i}+b_{j}-2 \\ a_{i}-1\end{array}\right) \not \equiv 0 \bmod p$ for $1 \leq i \leq r, 1 \leq j \leq s$.

Then for any shift-register sequences $\sigma$ and $\tau$ in $\mathbb{F}_{q}$ with $\sigma \in M(f)$ and $\tau \in$ $M(g)$, the product sequence $\sigma \tau$ is a shift-register sequence in $\mathbb{F}_{q}$ with minimal polynomial

$$
\prod_{\substack{1 \leq i \leq r \\ 1 \leq j \leq .}}\left(x-\alpha_{i} \beta_{j}\right)^{a_{i}+b,-1} \in \mathbb{F}_{q}[x] .
$$


Corollary 1. Under the conditions of Theorem 1, the sequence $\sigma \tau$ has linear complexity $L=s \operatorname{deg}(f)+r \operatorname{deg}(g)-r s$, which can be written as

$$
L=\operatorname{deg}(f) \operatorname{deg}(g)-(\operatorname{deg}(f)-r)(\operatorname{deg}(g)-s) .
$$

For the proof of Theorem 1 we need three auxiliary results. Lemma 2 below is taken from [4, Theorem 6.57].

Lemma 1. Let $F$ be an arbitrary field and $\alpha$ a nonzero element of $F$. Then for all integers $c, t \geq 0$ we have

$$
\left(\left(\begin{array}{c}
n+c \\
t
\end{array}\right) \alpha^{n}\right)_{n=0}^{\infty} \in M_{F}\left((x-\alpha)^{t+1}\right)
$$

Proof. We assign to the sequence $\left(\left(\begin{array}{c}n+c \\ t\end{array}\right) \alpha^{n}\right)_{n=0}^{\infty}$ its generating function

$$
G(x)=\sum_{n=0}^{\infty}\left(\begin{array}{c}
n+c \\
t
\end{array}\right) \alpha^{n} x^{-n-1}
$$

in the sense of Niederreiter [7]. Then

$$
\begin{aligned}
G(x) & =\sum_{n=\max (c, t)}^{\infty}\left(\begin{array}{l}
n \\
t
\end{array}\right) \alpha^{n-c} x^{-n+c-1} \\
& =\sum_{n=t}^{\infty}\left(\begin{array}{l}
n \\
t
\end{array}\right) \alpha^{n-c} x^{-n+c-1}-\sum_{n=t}^{\max (c, t)-1}\left(\begin{array}{l}
n \\
t
\end{array}\right) \alpha^{n-c} x^{-n+c-1} \\
& =\frac{\alpha^{t-c} x^{c}}{(x-\alpha)^{t+1}}-h(x)
\end{aligned}
$$

for some polynomial $h \in F[x]$. Thus $G(x)$ is a rational function which in its reduced form has the denominator $(x-\alpha)^{t+1}$, and so the result follows from [7, Lemma 2].

Lemma 2. Let $F$ be a finite field. If $\sigma_{i} \in M_{F}\left(h_{i}\right)$ for $1 \leq i \leq k$ and the monic polynomials $h_{1}, \ldots, h_{k} \in F[x]$ are pairwise relatively prime, then $\sigma_{1}+\cdots+\sigma_{k} \in$ $M_{F}\left(h_{1} \cdots h_{k}\right)$.

Lemma 3. Let $F$ be an arbitrary field of characteristic $p$, let $\alpha, \beta \in F$ with $\alpha \beta \neq 0$, and let $a$ and $b$ be positive integers. Let $\sigma$ and $\tau$ be shift-register sequences in $F$ with minimal polynomials $(x-\alpha)^{a}$ and $(x-\beta)^{b}$, respectively. Then $\sigma \tau$ is always in $S_{F}\left((x-\alpha \beta)^{a+b-1}\right)$, and $\sigma \tau \in M_{F}\left((x-\alpha \beta)^{a+b-1}\right)$ if and only if $\left(\begin{array}{c}a+b-2 \\ a-1\end{array}\right) \not \equiv 0 \bmod p$.

Proof. By Lemma 1,

$$
\psi_{i}:=\left(\left(\begin{array}{c}
n \\
i
\end{array}\right) \alpha^{n}\right)_{n=0}^{\infty} \in M_{F}\left((x-\alpha)^{i+1}\right) \quad \text { for } 0 \leq i \leq a-1 .
$$


It follows that the sequences $\psi_{0}, \ldots, \psi_{a-1}$ are linearly independent over $F$ and thus form a basis of the $a$-dimensional vector space $S_{F}\left((x-\alpha)^{a}\right)$. Hence every $\sigma \in S_{F}\left((x-\alpha)^{a}\right)$ can be uniquely represented in the form

$$
\sigma=c_{0} \psi_{0}+\cdots+c_{a-1} \psi_{a-1} \quad \text { with all } c_{i} \in F,
$$

and we have $\sigma \in M_{F}\left((x-\alpha)^{a}\right)$ if and only if $c_{a-1} \neq 0$. Again by Lemma 1,

$$
\omega_{j}:=\left(\left(\begin{array}{c}
n+j \\
j
\end{array}\right) \beta^{n}\right)_{n=0}^{\infty} \in M_{F}\left((x-\beta)^{j+1}\right) \quad \text { for } 0 \leq j \leq b-1 .
$$

Thus, the sequences $\omega_{0}, \ldots, \omega_{b-1}$ form a basis of $S_{F}\left((x-\beta)^{b}\right)$, and every $\tau \in$ $M_{F}\left((x-\beta)^{b}\right)$ has a unique representation

$$
\tau=d_{0} \omega_{0}+\cdots+d_{b-1} \omega_{b-1} \quad \text { with all } d_{j} \in F \text { and } d_{b-1} \neq 0 .
$$

If $\sigma \in M_{F}\left((x-\alpha)^{a}\right)$ and $\tau \in M_{F}\left((x-\beta)^{b}\right)$, then from (1) and (2) we get

$$
\sigma \tau=\sum_{\substack{0 \leq i \leq a-1 \\ 0 \leq j \leq b-1}} c_{i} d_{j} \psi_{i} \omega_{j}
$$

and so $\sigma \tau$ is a linear combination of product sequences of the form $\psi_{i} \omega_{j}$. The terms of $\psi_{i} \omega_{j}$ are

$$
\left(\begin{array}{c}
n \\
i
\end{array}\right)\left(\begin{array}{c}
n+j \\
j
\end{array}\right) \alpha^{n} \beta^{n}, \quad n=0,1, \ldots
$$

Now for all $i, j, n \geq 0$ we have

$$
\left(\begin{array}{c}
n \\
i
\end{array}\right)\left(\begin{array}{c}
n+j \\
j
\end{array}\right)=\left(\begin{array}{c}
i+j \\
i
\end{array}\right)\left(\begin{array}{c}
n+j \\
i+j
\end{array}\right)
$$

Thus, we can write the terms of $\psi_{i} \omega_{j}$ as

$$
\left(\begin{array}{c}
i+j \\
i
\end{array}\right)\left(\begin{array}{c}
n+j \\
i+j
\end{array}\right)(\alpha \beta)^{n}, \quad n=0,1, \ldots
$$

where the first factor is independent of $n$. Consequently, $\psi_{i} \omega_{j}$ is either the zero sequence, namely if $\left(\begin{array}{c}i+j \\ i\end{array}\right) \equiv 0 \bmod p$, or by Lemma 1 we have $\psi_{i} \omega_{j} \in M_{F}((x-$ $\left.\alpha \beta)^{i+j+1}\right)$ if $\left(\begin{array}{c}i+j \\ i\end{array}\right) \not \equiv 0 \bmod p$. From (3) it follows that $\sigma \tau \in S_{F}\left((x-\alpha \beta)^{a+b-1}\right)$. Furthermore, since $c_{a-1} d_{b-1} \neq 0$, we have $\sigma \tau \in M_{F}\left((x-\alpha \beta)^{a+b-1}\right)$ if and only if $\psi_{a-1} \omega_{b-1}$ is not the zero sequence, i.e., if and only if $\left(\begin{array}{c}a+b-2 \\ a-1\end{array}\right) \not \equiv 0 \bmod p$.

Proof of Theorem 1. Let $E$ again be the splitting field of $f g$ over $\mathbb{F}_{q}$. Then the sequences $\sigma \in M(f)$ and $\tau \in M(g)$ can be viewed as shift-register sequences in $E$. They have representations

$$
\begin{aligned}
& \sigma=\sigma_{1}+\cdots+\sigma_{r}, \\
& \tau=\tau_{1}+\cdots+\tau_{s},
\end{aligned}
$$


where the $\sigma_{i}$ and $\tau_{j}$ are shift-register sequences in $E$ with $\sigma_{i} \in M_{E}\left(\left(x-\alpha_{i}\right)^{a_{i}}\right)$ for $1 \leq i \leq r$ and $\tau_{j} \in M_{E}\left(\left(x-\beta_{j}\right)^{b_{j}}\right)$ for $1 \leq j \leq s$. It follows that

$$
\sigma \tau=\sum_{\substack{1 \leq i \leq r \\ 1 \leq j \leq s}} \sigma_{i} \tau_{j}
$$

By Lemma 3 and condition (ii) we have $\sigma_{i} \tau_{j} \in M_{E}\left(\left(x-\alpha_{i} \beta_{j}\right)^{a_{i}+b_{j}-1}\right)$. Since the $\alpha_{i} \beta_{j}$ are distinct by condition (i), we obtain from Lemma 2 that $\sigma \tau$, considered as a shift-register sequence in $E$, has the minimal polynomial

$$
h(x)=\prod_{\substack{1 \leq i \leq r \\ 1 \leq j \leq}}\left(x-\alpha_{i} \beta_{j}\right)^{a_{i}+b_{j}-1} .
$$

But from $f, g \in \mathbb{F}_{q}[x]$ we get

$$
h(x)^{q}=\prod_{\substack{1 \leq i \leq r \\ 1 \leq j \leq s}}\left(x^{q}-\alpha_{i}^{q} \beta_{j}^{q}\right)^{a_{i}+b_{j}-1}=\prod_{\substack{1<i \leq r \\ 1 \leq j \leq s}}\left(x^{q}-\alpha_{i} \beta_{j}\right)^{a_{i}+b_{j}-1}=h\left(x^{q}\right),
$$

and so $h \in \mathbb{F}_{q}[x]$. Consequently, $h$ is the minimal polynomial of $\sigma \tau$ as a shiftregister sequence in $\mathbb{F}_{q}$.

Remark 1. Consider the special case of Theorem 1 in which one of $f$ and $g$ has only simple roots. We then have $\operatorname{deg}(f)-r=0$ or $\operatorname{deg}(g)-s=0$. We assume again (i), and we note that (ii) is automatically satisfied since $\left(\begin{array}{c}a_{i}+b_{j}-2 \\ a_{1}-1\end{array}\right)=1$ for $1 \leq i \leq r, 1 \leq j \leq s$. It follows from Corollary 1 that the linear complexity $L$ of the product sequence $\sigma \tau$ is then $L=\operatorname{deg}(f) \operatorname{deg}(g)$. Thus, this special case of Theorem 1 yields a result of Herlestam [3] which says that under the indicated conditions the linear complexity of $\sigma \tau$ is equal to the product of the linear complexities of $\sigma$ and $\tau$ (compare with [3, Corollary 3.5]).

Remark 2. The congruence of Lucas [5] for binomial coefficients (see also [6] for a recent proof) implies that

$$
\left(\begin{array}{c}
a+b-2 \\
a-1
\end{array}\right) \neq 0 \bmod p \Leftrightarrow A_{m}+B_{m}<p \text { for all } m
$$

where the $A_{m}$ and $B_{m}$ are the digits in the $p$-ary expansion of $a-1$ and $b-1$, respectively, i.e.,

$$
a-1=\sum_{m \geq 0} A_{m} p^{m} \quad \text { and } \quad b-1=\sum_{m \geq 0} B_{m} p^{m}
$$

with $0 \leq A_{m}, B_{m}<p$ for all $m$. The connection with the operation $\vee$ introduced by Zierler and Mills [10] for positive integers $a$ and $b$ is given by

$$
\left(\begin{array}{c}
a+b-2 \\
a-1
\end{array}\right) \not \equiv \bmod p \Leftrightarrow a \vee b=a+b-1 \text {. }
$$


The fact that the condition (ii) in Theorem 1 is needed can be shown by the following simple example. Take $\alpha, \beta \in \mathbb{F}_{q}$ with $\alpha \beta \neq 0$ and integers $a, b \geq 1$ with $\left(\begin{array}{c}a+b-2 \\ a-1\end{array}\right) \equiv 0 \bmod p$. Then Lemma 3 shows that the conclusion of Theorem 1 is not valid for $\sigma \in M\left((x-\alpha)^{a}\right)$ and $\tau \in M\left((x-\beta)^{b}\right)$. In extreme cases such as the one considered in Theorem 2 below, the product sequence $\sigma \tau$ can even become the zero sequence.

Theorem 2. Let $f, g \in \mathbb{F}_{q}[x]$ be nonconstant monic polynomials with $f(0) \neq 0$ and $g(0) \neq 0$ and with canonical factorizations

$$
f=\prod_{i=1}^{k} f_{i}^{a_{1}} \quad \text { and } \quad g=\prod_{j=1}^{l} g_{j}^{b j}
$$

in $\mathbb{F}_{q}[x]$. Suppose the exponents $a_{i}$ and $b_{j}$ are such that $\left(\begin{array}{c}a_{i}+b_{j}-2 \\ a_{i}-1\end{array}\right) \equiv 0 \bmod p$ for $1 \leq i \leq k, 1 \leq j \leq l$. Then there exist shift-register sequences $\sigma \in M(f)$ and $\tau \in M(g)$ in $\mathbb{F}_{q}$ such that $\sigma \tau$ is the zero sequence.

Proof. For a finite extension field $F$ of $K=\mathbb{F}_{q}$ let $\operatorname{Tr}_{F / K}$ be the trace function from $F$ onto $K$ (compare with [4, Definition 2.22]). For $1 \leq i \leq k$ let $\sigma_{i}$ be the sequence with terms

$$
\left(\begin{array}{c}
n \\
a_{i}-1
\end{array}\right) \operatorname{Tr}_{F_{1} / K}\left(\alpha_{i}^{n}\right), \quad n=0,1, \ldots,
$$

where $\alpha_{i}$ is a root of $f_{i}$ and $F_{i}=\mathbb{F}_{q}\left(\alpha_{i}\right)$. It follows from Lemma 1 and Lemma 2 that $\sigma_{i} \in M\left(f_{i}^{a_{i}}\right)$ for $1 \leq i \leq k$, and so $\sigma:=\sigma_{1}+\cdots+\sigma_{k} \in M(f)$ by Lemma 2 . Similarly, we define for $1 \leq j \leq l$ the sequence $\tau_{j}$ with terms

$$
\left(\begin{array}{c}
n+b_{j}-1 \\
b_{j}-1
\end{array}\right) \operatorname{Tr}_{E_{j} / K}\left(\beta_{j}^{n}\right), \quad n=0,1, \ldots
$$

where $\beta_{j}$ is a root of $g_{j}$ and $E_{j}=\mathbb{F}_{q}\left(\beta_{j}\right)$. Then $\tau_{j} \in M\left(g_{j}^{b_{j}}\right)$ for $1 \leq j \leq l$ and $\tau:=\tau_{1}+\cdots+\tau_{l} \in M(g)$. By (4) and the given hypothesis, for all $1 \leq i \leq k$, $1 \leq j \leq l$ we have

$$
\left(\begin{array}{c}
n \\
a_{i}-1
\end{array}\right)\left(\begin{array}{c}
n+b_{j}-1 \\
b_{j}-1
\end{array}\right)=\left(\begin{array}{c}
a_{i}+b_{j}-2 \\
a_{i}-1
\end{array}\right)\left(\begin{array}{c}
n+b_{j}-1 \\
a_{i}+b_{j}-2
\end{array}\right) \equiv 0 \bmod p
$$

and so $\sigma_{i} \tau_{j}$ is the zero sequence. This implies that $\sigma \tau=\sum_{i=1}^{k} \sum_{j=1}^{l} \sigma_{i} \tau_{j}$ is the zero sequence.

We recall a definition from Zierler and Mills [10]. If $f$ and $g$ are nonconstant monic polynomials over $\mathbb{F}_{q}$ with simple roots, then $f \vee g \in \mathbb{F}_{q}[x]$ is the monic polynomial whose roots are exactly the distinct elements of the form $\alpha \beta$, where $\alpha$ is a root of $f$ and $\beta$ a root of $g$ in the splitting field of $f g$ over $\mathbb{F}_{q}$. The following result can be viewed as an extension of Theorem 2 . 
Theorem 3. Let $f, g \in \mathbb{F}_{q}[x]$ be nonconstant monic polynomials with $f(0) \neq 0$ and $g(0) \neq 0$ and with canonical factorizations

$$
f=\prod_{i=1}^{k} f_{i}^{a_{1}} \quad \text { and } \quad g=\prod_{j=1}^{l} g_{j}^{b_{j}}
$$

in $\mathbb{F}_{q}[x]$. Then there exist shift-register sequences $\sigma \in M(f)$ and $\tau \in M(g)$ in $\mathbb{F}_{q}$ such that $\sigma \tau$ has

$$
\operatorname{lcm}\left\{\left(f_{i} \vee g_{j}\right)^{a_{i}+b_{j}-1}: 1 \leq i \leq k, 1 \leq j \leq l,\left(\begin{array}{c}
a_{i}+b_{j}-2 \\
a_{i}-1
\end{array}\right) \not \equiv 0 \bmod p\right\}
$$

as a characteristic polynomial.

Proof. For $1 \leq i \leq k$ and $1 \leq j \leq l$ define the sequences $\sigma_{i}$ and $\tau_{j}$ as in the proof of Theorem 2, and put $\sigma=\sigma_{1}+\cdots+\sigma_{k} \in M(f)$ and $\tau=\tau_{1}+\cdots+\tau_{l} \in M(g)$. Then

$$
\sigma \tau=\sum_{\substack{1 \leq i \leq k \\ 1 \leq j \leq 1}} \sigma_{i} \tau_{j} .
$$

In the proof of Theorem 2 it was shown that $\sigma_{i} \tau_{j}$ is the zero sequence for all $(i, j)$ with $\left(\begin{array}{c}a_{1}+b_{j}-2 \\ a_{1}-1\end{array}\right) \equiv 0 \bmod p$. Thus we can write

$$
\sigma \tau=\sum_{(i, j) \in I} \sigma_{i} \tau_{j}
$$

with $I=\left\{(i, j): 1 \leq i \leq k, 1 \leq j \leq l,\left(\begin{array}{c}a_{1}+b_{j}-2 \\ a_{1}-1\end{array}\right) \not \equiv 0 \bmod p\right\}$. Since $\sigma_{i} \in S\left(f_{i}^{a_{1}}\right)$ and $\tau_{j} \in S\left(g_{j}^{b_{j}}\right)$, it follows from Zierler and Mills [10] that $\sigma_{i} \tau_{j}$ has $\left(f_{i} \vee g_{j}\right)^{a, \vee b}$, as a characteristic polynomial. By Remark 2 we have $a_{i} \vee b_{j}=a_{i}+b_{j}-1$ for $(i, j) \in I$. Hence we obtain from (5) that a characteristic polynomial of $\sigma \tau$ is given by the least common multiple of the polynomials $\left(f_{i} \vee g_{j}\right)^{a_{1}+b,-1}$, $(i, j) \in I$.

Finally, we combine Theorem 1 with a result of Rueppel and Staffelbach [9].

Corollary 2. Let $f \in \mathbb{F}_{q}[x]$ be a monic irreducible polynomial with $f(0) \neq 0$ and let $g \in \mathbb{F}_{q}[x]$ be a nonconstant monic polynomial with $g(0) \neq 0$ and simple roots. Define $R=\operatorname{ord}(f) / \operatorname{gcd}(\operatorname{ord}(f), \operatorname{ord}(g))$. Suppose the multiplicative order of $q$ modulo $R$ equals $\operatorname{deg}(f)$. Let $a$ and $b$ be two positive integers with $\left(\begin{array}{c}a+b-2 \\ a-1\end{array}\right) \not \equiv$ $0 \bmod p$. Then for any shift-register sequences $\sigma$ and $\tau$ in $\mathbb{F}_{q}$ with $\sigma \in M\left(f^{a}\right)$ and $\tau \in M\left(g^{b}\right)$, the product sequence $\sigma \tau$ has the minimal polynomial $(f \vee g)^{a+b-1}$ and so the linear complexity $(a+b-1) \operatorname{deg}(f) \operatorname{deg}(g)$.

Proof. In [9] Rueppel and Staffelbach have shown that under the conditions in the corollary the products of a root of $f$ and a root of $g$ are distinct. Therefore the polynomials $f^{a}$ and $g^{b}$ satisfy the hypotheses of Theorem 1 . 


\section{References}

[1] Golić, J. Dj.: On the linear complexity of functions of periodic GF $(q)$ sequences, IEEE Trans. Inform. Theory 35, 69-75 (1989).

[2] Göttfert, R.: Produkte von Schieberegisterfolgen, Ph.D. Dissertation, Univ. of Vienna, 1993.

[3] Herlestam, T.: On functions of linear shift register sequences, Advances in Cryptology - EUROCRYPT ' 85 (F. Pichler, ed.), Lecture Notes in Computer Science, vol. 219, pp. 119-129, Springer-Verlag, Berlin, 1986.

[4] Lidl, R., and Niederreiter, H.: Introduction to Finite Fields and Their Applications, Cambridge University Press, Cambridge, 1986.

[5] Lucas, E.: Sur les congruences des nombres eulériens et des coefficients différentiels des fonctions trigonométriques, suivant un module premier, Bull. Soc. Math. France 6, 49-54 (1878).

[6] McIntosh, R.J.: A generalization of a congruential property of Lucas, Amer. Math. Monthly 99, 231-238 (1992).

[7] Niederreiter, H.: Sequences with almost perfect linear complexity profile, Advances in Cryptology - EUROCRYPT '87 (D. Chaum and W. L. Price, eds.), Lecture Notes in Computer Science, vol. 304, pp. 37-51, SpringerVerlag, Berlin, 1988.

[8] Rueppel, R.A.: Stream ciphers, Contemporary Cryptology: The Science of Information Integrity (G.J. Simmons, ed.), pp. 65-134, IEEE Press, New York, 1992.

[9] Rueppel, R.A., and Staffelbach, O. J.: Products of linear recurring sequences with maximum complexity, IEEE Trans. Inform. Theory 33, 124-131 (1987).

[10] Zierler, N., and Mills, W.H.: Products of linear recurring sequences, $J$. Algebra 27, 147-157 (1973). 